\title{
Die Beeinflussung der Atmungsintensität pflanzlicher Gewebe durch eine Belichtung in fluoreszierenden Farbstofflösungen
}

\author{
Von Hubert Ziegler \\ Aus dem Botanischen Institut der Universität München \\ (Z. Naturforschg. 5 b, 345-350 [1950]; eingegangen am 24. August 1950)
}

\begin{abstract}
1. Der Sauerstoffverbrauch von Pflanzengewebe in belichteten Lösungen von Fluoreszein-K, Eosin-w-gelbl. und Jodeosin zeigt gegenüber den Dunkelwerten eine Steigerung von $20-50 \%$.

2. Bei dieser verstärkten Sauerstoffaufnahme handelt es sich um eine Intensivierung der Atmung; der respiratorische Quotient bleibt unverändert auf dem Wert 1.
\end{abstract}

$\mathrm{B}$ elichtete Lösungen fluoreszierender Substanzen beeinflussen eine Reihe von Wachstumsvorgängen und Reizerscheinungen der Organismen ${ }^{1}$. Da diese sog. photodynamischen Erscheinungen nach Tappeiner und J odlbauer ${ }^{2}$ nur bei Gegenwart von genügend freiem Sauerstoff auftreten, lag die Vermutung nahe, daß es sich dabei um die Wirkungen photooxydativer Prozesse handelte. Diese Annahme wurde durch die Untersuchungen Noa ck s ${ }^{3}$ bestätigt, der nachwies, daß die photodynamische Wirkung von Eosin auf Paramaecien durch einen geeigneten Sauerstoffacceptor, z. B. Natriumsulfit, vollständig aufgehoben und durch Sauerstoffüberträger, z. B. Mangansalze, stark beschleunigt werden kann. Noack ${ }^{4}$ stellte ferner fest, daß auch der wichtigste fluoreszierende Pflanzenfarbstoff, das Chlorophyll, sowohl in vitro als auch in der lebenden Zelle sauerstoffaktivierende Eigenschaften besitzt.

Um eine Oxydation des Serumeiweißes durch die photodynamische Wirkung belichteten Hämatoporphyrins, eines Abbauproduktes des Blutfarbstoffes, handelt es sich nach $\mathrm{G}$ aff $\mathrm{r}$ o $\mathrm{n}^{5}$ bei der Porphyrie, einer seltenen Erkrankung des menschlichen Organismus.

Eine photooxydative Wirksamkeit belichteter, fluoreszierender Farbstoffe ließ sich endlich auch in zahlreichen Modellversuchen feststellen ${ }^{6}$.

Steht somit die Beeinflußbarkeit der Oxydationsprozesse in lebenden und toten Systemen durch das

1 P. M e t z n e r, Biochem. Z. 101, 33 [1919]; 113, 145 [1921]; Ber. dtsch. bot. Ges. 41, 268 [1923]; H. T a k e d a, Sci. Rep. Tôhoku Univ. 4, 557 [1929]; P. B oy s en-Jens en, Planta 22, 404 [1934]; L. S te inke, Planta 30, 757 [1940]; H. Z i e g l e r, Planta (im Druck).

$2 \mathrm{H}$. von Tappeiner u. F. J od l b a e r, Dtsch. Arch. klin. Med. 82 [1905]; Biochem. Z. 12, 290 [1908].

3 K. N o a c k, Z. Bot. 12, 273 [1920].
Licht nach einer Sensibilisierung mit fluoreszierenden Farbstoffen außer Zweifel, so fehlte doch bisher eine eingehendere quantitative Erfassung dieser Wirkungen durch eine Messung des veränderten Sauerstoffverbrauches. Sehr spärlich sind auch die Angaben über die Beziehungen der photodynamischen Erscheinungen $\mathrm{zu}$ dem wichtigsten sauerstoffverbrauchenden Prozeß eines lebenden Systems, zur Atmung. $\mathrm{Ge} \beta n$ e ${ }^{7}$ fand, daß die bei Elodea-Sprossen für das Assimilationssystem festgestellte photodynamische Rhodaminschädigung ${ }^{8}$ sich nicht auf das Atmungssystem erstreckt. G e $\beta \mathrm{n}$ er erhielt bei Sprossen, die 3 Stdn. mit Rhodamin 1:1000 gefärbt worden waren, eine Atmungsintensität von $98 \%$, bei ebensolange im Dunkeln gefärbten von $126 \%$ derjenigen ungefärbter Sprosse. S c h ülk e ${ }^{9}$ wies nach, daß die von ihr bei Hefe nach Zusatz von Porphyrinen in geringer Konzentration erhaltene Atmungssteigerung und die bei höherer Konzentration festgestellte Atmungshemmung durch eine Bestrahlung mit Ultraviolettlicht verstärkt werden.

Eine eingehendere Untersuchung der Wirkung belichteter, fluoreszierender Farbstofflösungen auf die Sauerstoffaufnahme pflanzlicher Gewebe unter besonderer Berücksichtigung einer etwaigen Beeinflussung der Atmung, wie sie ähnlich Kostytschew ${ }^{10}$ schon 1924 forderte, schien von Interesse und war das Ziel der nachstehend beschriebenen Versuche.

4 K. N o a c k, Naturwiss. 14, 383 [1926].

5 H. G a f f r o n, Naturwiss. 13, 859 [1925].

6 K. N o a c k, Naturwiss. 14, 383 [1926].

7 Fr. Ge $\beta$ n e r, Planta 32, 1 [1941].

8 A. Pirs on u. F. A l b e rts, Protoplasma 35, 131 [1941].

9 H. S c h ülk e, Biochem. Z. 311, 146 [1941/42].

10 .S. K o s t y t s c h ew, Pflanzenatmung, Berlin 1924. 


\section{Die Versuche}

\section{Material und Methode}

Zur Klarstellung der Beziehungen der photodynamischen Oxydationswirkung zur Atmung kamen nur nichtassimilierende Objekte in Betracht; verwendet wurden Wurzeln von Daucus Carota, Petroselinum hortense und Cochlearia Armoracia, deren Nährstoffreichtum eine gleichmäßige Atmung auch bei längerer Versuchsdauer gewährleistete, und die chlorophyllfreien Sprosse von Asparagus officinalis.

Zur Messung des Sauerstoffverbrauchs diente die manometrische Methode, wobei das Verfahren im einzelnen dem von $\mathrm{K}$ andler ${ }^{11}$ benützten weitgehend entsprach. Die Vorzüge dieser Arbeitsweise gerade für die Bearbeitung des oben skizzierten Problems liegen in der Möglichkeit der Registrierung auch geringerer Schwankungen über längere Zeit und eines öfteren Wechsels von Belichtung und Verdunkelung bei weitestgehender Temperaturkonstanz.

Als Lichtquelle wurden zwei Bergmann-Röhren verwendet, die sich unter dem Boden des Glasthermostaten befanden, dessen Seitenwände schwarz gestrichen waren. Die Lichtintensität belief sich in Höhe der Versuchsgefäße auf etwa 1000 Lux.

Die Temperatur betrug durchweg $29^{\circ} \mathrm{C}$; die Wärmezufuhr durch die Belichtung wurde durch eine Kühlschlange ausgeglichen. Die Temperaturschwankungen beliefen sich auf $\pm 0,1^{\circ} \mathrm{C}$.

Die Gefäße wurden mit $3 \mathrm{~cm}^{3} 1 /{ }_{30}-m$. Phosphatpuffers vom $p_{\mathrm{H}} 6,2$ beschickt, denen bei den Farbstoffversuchen eine der angegebenen Molarität entsprechende Menge des Farbstoffes zugegeben wurde. In dieser Lösung lagen die Gewebeteile. Bei der Messung der Sauerstoffaufnahme kamen zur Absorption des $\mathrm{CO}_{2}$ in die Ansatzbirne $0,3 \mathrm{~cm}^{3}$ 1- $n$. Kalilauge. Die Absorptionsfläche wurde durch Einlegen eines F'iltrierpapierstreifens vergrößert.

Die erhaltenen Werte des Sauerstoffverbrauches wurden nicht auf Trocken- oder Frischgewicht bezogen, da hier erfahrungsgemäß die Fehlerquelle ziemlich groß ist. Als Vergleichsgrundlage diente in allen Fällen eine einstündige Leeratmung. Es wurden dabei zunächst alle Gefäße nur mit Material gleichen Frischgewichtes und mit der Pufferlösung versehen, die Sauerstoffaufnahme kontrolliert und dann erst der Farbstoff zugegeben. Nur Versuchsreihen, deren Leeratmungswerte nicht mehr als $5 \%$ auseinanderlagen, wurden weiterverwendet. Der Bezugs-

11 O. K a nd le r, Z. Naturforschg. 5 b, 203 [1950]. wert war stets der Wert des späteren Vergleichsgefäßes, das während der ganzen Versuchsdauer nur mit dem Material und dem Puffer beschickt wurde.

Im Gasraum befand sich durchweg Luft. Zur Korrektur der Temperatur- und Luftdruckschwankungen diente ein Thermobarometer.

Die Gefäßkonstanten betrugen 1,15-1,25. Jeder abgelesene $\mathrm{mm}$ Druckdifferenz entsprach demnach $1,15-1,25 \mathrm{~mm}^{3}$ verbrauchten Sauerstoffes. Die Ablesung war auf $0,5 \mathrm{~mm}^{3}$ genau und erfolgte stets in halbstündigem Abstand.

Nach Beendigung jedes Versuches wurde das $p_{\mathrm{H}}$ der Versuchsflüssigkeiten mittels eines Potentiometers mit Chinhydronelektrode kontrolliert. Die Abweichungen vom Ausgangs- $p_{\mathrm{H}}$ waren stets gering: sie betrugen höchstens $\pm 0,2$.

Das Material wurde mit einem Mikrotom geschnitten. Die Schnittdicke war daher weitgehend gleichmäßig (etwa 0,15 mm). Die Grenzschnittdicke, die nach Dickens ${ }^{12}$ für pflanzliche Gewebe in Luft unter $0,5 \mathrm{~mm}$ liegt, wurde also sicher nicht erreicht.

\section{B lindversuche}

Es war zunächst zu prüfen, ob die gepufferten Farbstofflösungen ohne Pflanzenmaterial irgendeine Sauerstoffaufnahme, sei es im Dunkeln oder im Licht, zeigten. Es wurde diesbezüglich untersucht Fluoreszein- $\mathrm{K}$ in den molaren Konzentrationen: $2,5 \times 10^{-3}$, $5 \times 10^{-4}$ und $5 \times 10^{-5}$, Eosin $1 \times 10^{-3}, 1 \times 10^{-4}$, $2,5 \times 10^{-4}$ und $2,5 \times 10^{-5}$ und Jodeosin $1 \times 10^{-3}$ und $1 \times 10^{-5}$. Eine Sauerstoffaufnahme konnte in keinem Falle festgestellt werden.

\section{Versuche mit Wurzeln}

a) Die Dunkelwirkung der Farbstoffe

In Fluoreszeinlösungen verschiedener Konzentration ließ unbelichtetes Wurzelgewebe von Petroselinum hortense eine im Vergleich mit den entsprechenden Werten im ungefärbten Puffer leicht gehemmte Sauerstoffaufnahme erkennen; der Betrag der Hemmung zeigte dabei keine strenge Proportionalität zu der Farbstoffkonzentration (Abb. 1 und 2).

Ein etwas anderes Bild geben die Kurven für die Sauerstoffaufnahme von Petersilienwurzeln in unbelichteten Eosinlösungen verschiedener Konzentration (Abb. 3). Deutlich ist hier eine Hemmung ausgeprägt, deren Stärke der Konzentration der Lösung proportional ist. Bemerkenswert ist die leichte För-

12 B a m a n n-M y r b ä ck II, 2 c, S. 1339. 


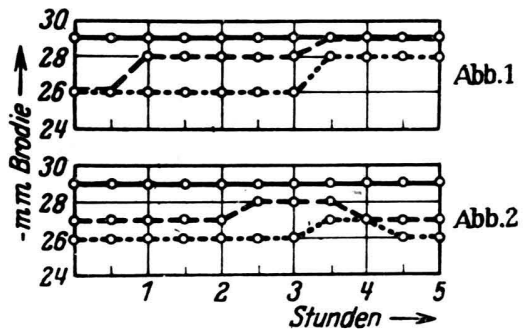

Abb. 1. Sauerstoffaufnahme unbelichteten Wurzelgewebes von Petroselinum hortense im Puffer in Fluoreszein in Fluoreszein $5 \times 10^{-4} \mathrm{~mol}$. $2,5 \times 10^{-4} \mathrm{~mol}$. - - -

Abb. 2. Sauerstoffaufnahme unbelichteten Wurzelgewebes von Petroselinum hortense im Puffer in Fluoreszein $1 \times 10^{-3} \mathrm{~mol}$. in Fluoreszein $5 \times 10^{-3} \mathrm{~mol}$.

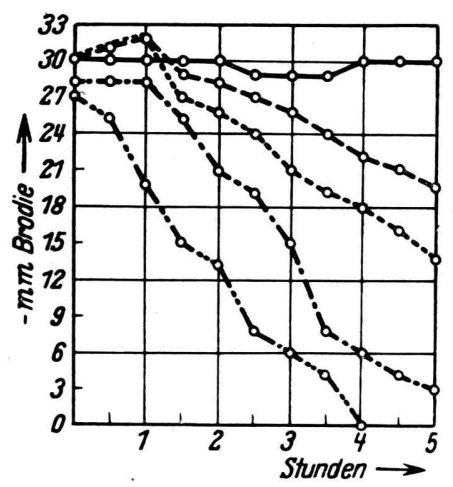

Abb. 3. Sauerstoffaufnahme unbelichteten Wurzelgewebes von Petroselinum hortense im Puffer
in Eosin
$1 \times 10^{-4} \mathrm{~mol}$ in Eosin
$1 \times 10^{-3} \mathrm{~mol}$
in Eosin
in Eosin
$2,5 \times 10^{-4}$ mol. . . . . -
$2,5 \times 10^{-3} \mathrm{~mol}$

derung bei schwächeren Konzentrationen zu Beginn der Einwirkung der Farbstofflösungen, die sich regelmäßig einstellte.

Bei Karottenwurzeln liegen die Verhältnisse sehr ähnlich; auch hier ist in unbelichtetem Fluoreszein eine leichte, in unbelichtetem Eosin eine starke Hemmung der Sauerstoffaufnahme festzustellen.

b) Die Lichtwirkung der Farbstoffe

Zur Beobachtung der Wirkung belichteter Lösungen fluoreszierender Farbstoffe auf die Sauerstoffaufnahme von Wurzelgewebe wurde zunächst während der gesamten Versuchsdauer belichtet und die Hälfte der Versuchsgefäße mit schwarzem Stoff umwickelt, so daß hier der Farbstoff im Dunkeln wirkte und damit eine Vergleichsgrundlage gegeben war. Die Abbildungen 4,5 und 6 geben die erhaltenen

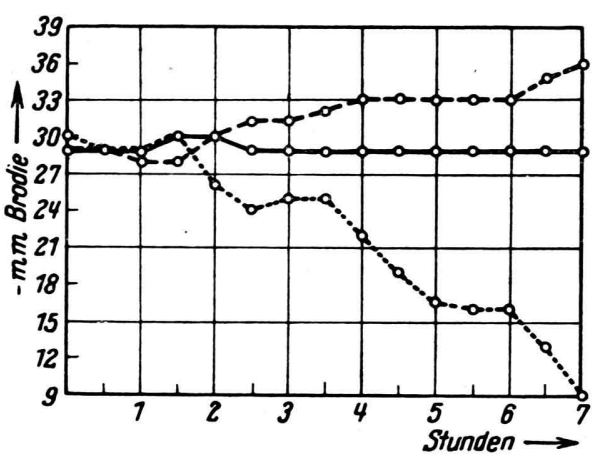

Abb. 4. Sauerstoffaufnahme desWurzelgewebes von Daucus Carota in Eosinlösungen der Konzentration 2,5 $\times 10^{-4} \mathrm{~mol}$. im Licht - - - - im Dunkeln - - in der belichteten Pufferlösung

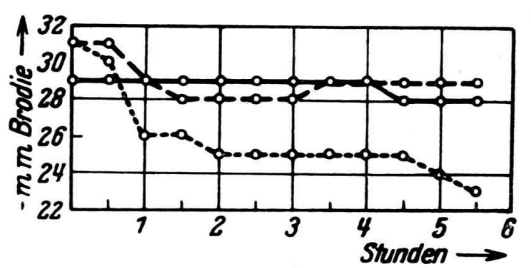

Abb. 5. Sauerstoffaufnahme desWurzelgewebes von Daucus Carota in Fluoreszeinlösungen der Konzentration $5 \times 10^{-4} \mathrm{~mol}$ im Licht _ _ im Dunkeln . . . . in der belichteten Pufferlösung

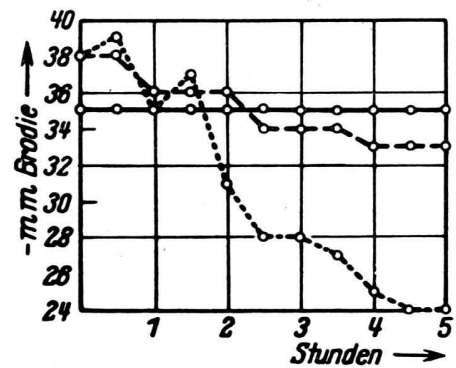

Abb. 6. Sauerstoffaufnahme des Wurzelgewebes von Petroselinum hortense in Eosinlösungen der Konzentration $1 \times 10^{-4} \mathrm{~mol}$.

im Licht _ _ - im Dunkeln ......

in der belichteten Pufferlösung

Werte wieder. Die Kurven zeigen eine beträchtliche Steigerung der Sauerstoffaufnahme in den belichteten Farbstofflösungen im Vergleich mit den Werten in den unbelichteten. Die Förderung ist durchweg in Eosin stärker ausgeprägt als in Fluoreszein, womit sich eine Parallele zu der photodynamischen Wirksamkeit dieser Farbstoffe abzeichnet. Die Intensivierung der Sauerstoffaufnahme kann so weit gehen, daß die im Dunkeln normalerweise einsetzende Hemmung überkompensiert wird und das Gewebe in den 


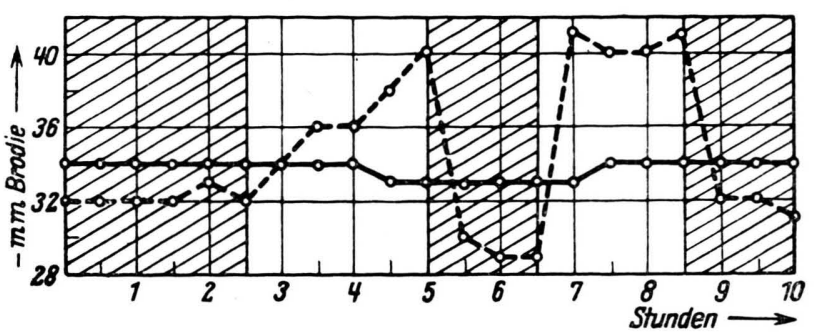

Abb. 7. Sauerstoffaufnahme des Wurzelgewebes von Cochlearia Armoracia in belichtetem und unbelichtetem Fluoreszein der Konzentration $2,5 \times 10^{-3} \mathrm{~mol}$. . . . und in Pufferlösung Schraffierte Bereiche dunkel.

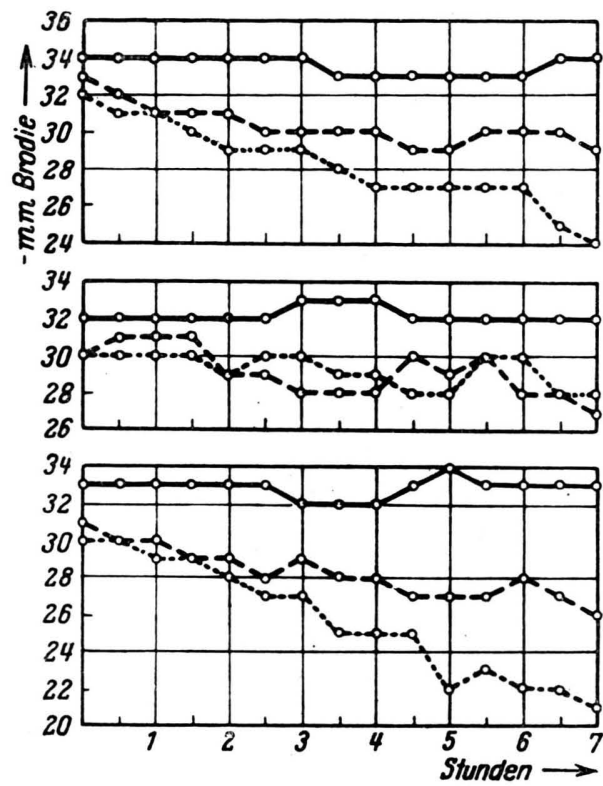

Abb. 8

Abb. 9

Abb. 8. Sauerstoftaufnahme unbelichteten Sproßgewebes von Asparagus officinalis in Eosinlösungen der Konzentrationen

$1 \times 10^{-3} \mathrm{~mol}$. - . - . 2, $2,5 \times 10^{-4} \mathrm{~mol}$. _ und im Puffer

Abb. 9. Sauerstoffaufnahme unbelichteten Sproßgewebes von Asparagus officinalis in Fluoreszeinlösungen der Konzentrationen

$2,5 \times 10^{-3} \mathrm{~mol}$. - . - $5 \times 10^{-4} \mathrm{~mol}$.

und im Puffer

Abb. 10. Sauerstoffaufnahme unbelichteten Sproßgewebes von Asparagus officinalis in Jodeosinlösungen der Konzentrationen

$1 \times 10^{-5} \mathrm{~mol}$. - . - $1 \times 10^{-6} \mathrm{~mol}$. und im Puffer

belichteten Farbstofflösungen eine höhere Sauerstoffaufnahme zeigt als im Vergleichspuffer ohne Farbstoffzusatz.

Das eben beschriebene Verfahren der Verdunkelung einzelner Gefäße ist insofern nicht als ideal zu bezeichnen, als die verschiedenen Absorptionsverhältnisse die Konstanz der Temperatur gefährden können. Da es aber immer nur zu einer Temperatursteigerung in den Dunkelgefäßen kommen kann, liegt die dadurch bedingte vermehrte Sauerstoffaufnahme so, daß der Wert der relativen Förderung der Sauerstoffaufnahme in den belichteten Farbstofflösungen höchstens zu niedrig, unbedingt aber reell ist.

Zur Erzielung eines von dieser möglichen Beeinflussung freien Ergebnisses wurden für eine Versuchsreihe, die mit Wurzelgewebe von Cochlearia Armoracia durchgeführt wurde, die Werte für die Sauerstoffaufnahme im Licht und im Dunkeln dadurch festgestellt, daß durch Ein- und Ausschalten der Beleuchtung die gesamten Versuchsgefäße abwechselnd belichtet und verdunkelt wurden. Abb. 7 zeigt das Versuchsergebnis, das ebenfalls klar die gesteigerte Sauerstoffaufnahme bei Belichtung vor Augen führt.

\section{Versuche mit Sprossen}

a) Die Dunkelwirkung der Farbstoffe

Die Sauerstoffaufnahme des Sproßgewebes von Asparagus officinalis ist in unbelichteten Lösungen von Fluoreszein, Eosin und Jodeosin verschiedener Konzentration ebenfalls merklich geschwächt. Diese Hemmung ist beim Fluoreszein am geringsten, beim Jodeosin am stärksten ausgeprägt und steigt bei Eosin und Jodeosin mit der Konzentration der Lösung (Abb. 8, 9, 10). Bemerkenswert ist weiter der im Vergleich mit den bei den Wurzeln erhaltenen Werten weit weniger ausgeprägte Abfall der Sauerstoffaufnahme in den Eosinlösungen.

b) Die Lichtwirkung der Farbstoffe

In den Versuchen, die der Feststellung der Sauerstoffaufnahme von Sproßgewebe in Lösungen belichteter fluoreszierender Farbstoffe dienen sollten, wurde durchweg das Verfahren einer abwechselnden Belichtung und Verdunkelung der gesamten Versuchsgefäße angewandt, wie es bei den letzten Wurzelversuchen beschrieben wurde.

Es zeigte sich, daß auch die Sauerstoffaufnahme des Sproßgewebes von Asparagus officinalis in Fluoreszein-, Eosin- und Jodeosinlösungen bei Belichtung eine Verstärkung erfährt (Abb.11, 12, 13, 14). Eine leichte Nachwirkung dieser Förderung in der Dunkelphase ist unverkennbar. In Abb. 13 ist das Erlöschen der Stimulationswirkung beim Unterschreiten einer bestimmten Konzentration zu erkennen. 


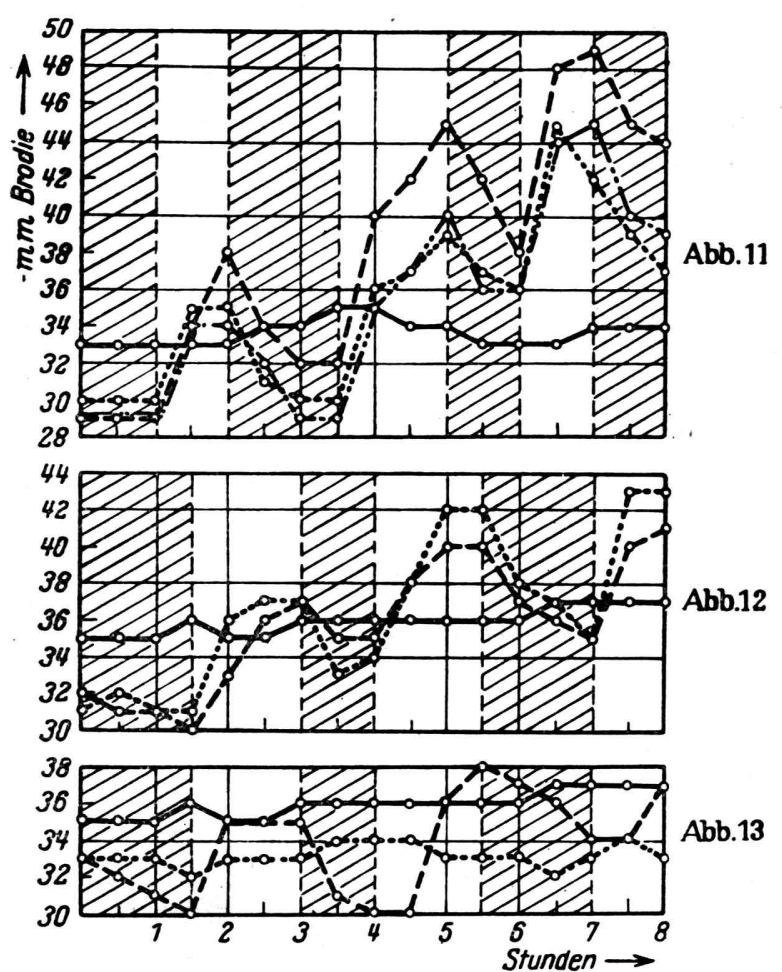

Abb. 11. Sauerstoffaufnahme des Sproßgewebes von Asparagus officinalis in belichteten und unbelichteten

Fluoreszeinlösungen der Konzentrationen

$2,5 \times 10^{-4} \mathrm{~mol}$. - - $5 \times 10^{-4} \mathrm{~mol}$. .........

$2,5 \times 10^{-3} \mathrm{~mol}$. - . . . - und im Puffer Schraffierte Bereiche dunkel.

Abb. 12. Sauerstoffaufnahme des Sproßgewebes von Asparagus officinalis in belichteten und unbelichteten Eosinlösungen der Konzentrationen

$$
\begin{gathered}
1 \times 10^{-3} \mathrm{~mol} .-\ldots 2,5 \times 10^{-4} \mathrm{~mol} . \\
\text { und im Puffer } \\
\text { Schraffierte Bereiche dunkel. }
\end{gathered}
$$

Abb. 13. Sauerstoffaufnahme des Sproßgewebes von Asparagus officinalis in belichteten und unbelichteten Eosinlösungen der Konzentrationen

$1 \times 10^{-4} \mathrm{~mol}$. - - $1 \times 10^{-5} \mathrm{~mol}$. - . - . und im Puffer

Schraffierte Bereiche dunkel.

Aus all diesen Versuchen geht klar hervor, daß der Sauerstoffverbrauch von chlorophyllfreiem Pflanzengewebe in den verwendeten Konzentrationen von Fluoreszein, Eosin und Jodeosin bei Belichtung beträchtlich gesteigert wird. Die Beträge der Förderung liegen im allgemeinen zwischen 20 und $50 \%$ des Dunkelverbrauches, wobei die Werte nur teilweise der Stärke der photodynamischen Wirkung der einzelnen Stoffe parallel gehen.

Hinzuweisen ist ferner darauf, daß der Sauerstoffverbrauch in den Kontrollgefäßen, in denen sich das

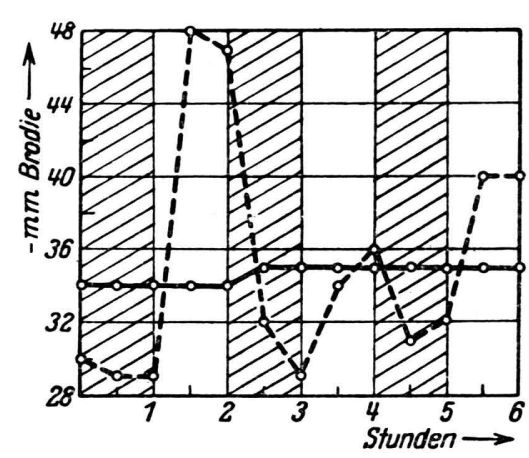

Abb. 14. Sauerstoffaufnahme des Sproßgewebes von Asparagus officinalis in belichteten und unbelichteten Jodeosin-

lösungen der Konzentration $1 \times 10^{-6} \mathrm{~mol}$. _ _ und im Puffer

Schraffierte Bereiche dunkel.

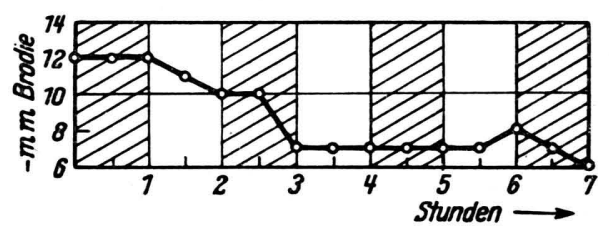

Abb. 15. Sauerstoffverbrauch des Sproßgewebes von Neottia nidus avis in Phosphatpufferlösung bei Belichtung und im Dunkeln.

Schraffierte Bereiche dunkel.

Material in der ungefärbten Pufferlösung befand, keinerlei gesetzmäßige Abhängigkeit von Belichtung und Verdunkelung zeigte, eine "Lichtatmung“ also nicht beobachtet werden konnte; sie war unter den Bedingungen der Versuche auch nicht zu erwarten ${ }^{13}$.

Schließlich ist noch die hervorragende Eignung von Asparagus-Sprossen für manometrische Atmungsbestimmungen hervorzuheben, bei denen nichtassimilierendes Sproßmaterial zur Anwendung kommen soll. Das Gewebe zeigt eine relativ intensive Atmung, keine Assimilation auch nach mehrstündiger Belichtung und ermöglicht außerdem infolge der besonderen Beschaffenheit der Sprosse außerordentlich dünne Schnitte.

Weit weniger eignen sich zu Atmungsversuchen Sprosse von Neottia nidus avis, da ihr Sauerstoffverbrauch sehr gering ist. Sie wurden daher nach einigen Vorversuchen nicht weiter für die Untersuchung der Wirkung belichteter Farbstofflösungen verwendet. Da sich aber hier die günstige Gelegenheit bot, die immer noch strittige Frage einer evtl. geringen Assimilation von Neottia ${ }^{14}$ mittels der empfindlichen manometri-

13 Vgl. C. Montfort u. G. Rosenstock, Z. Naturforschg. 5 b, 171 [1950]. 
schen Methode zu prüfen, wurden einige Belichtungsversuche angestellt. Abb. 15 gibt die Mittelwerte von 6 Gefäßen, die das Neottia-Sproßgewebe in einer $1 / 30^{-m}$. Phosphatpufferlösung vom $p_{\mathrm{II}} 6,2$ enthielten.

$\mathrm{Da}$ in allen Gefäßen wohl die durch die Atmung gebildete Kohlensäure durch Kalilauge absorbiert wurde, nicht aber der bei einer möglicherweise einsetzenden Assimilation entstehende Sauerstoff, würde sich das Einsetzen assimilatorischer Prozesse in der Kurve als ein Absinken des Sauerstoffverbrauches bei Belichtung zu erkennen geben, da ja dann das durch den Sauerstoffverbrauch entstehende Druckdefizit durch die Sauerstoffbildung verringert, aufgehoben oder sogar überkompensiert werden müßte. Das ist aber offenbar nicht der Fall; es kann daher gesagt werden, daß unter den Bedingungen des Versuches $\left(29^{\circ} \mathrm{C}, 1000\right.$ Lux Beleuchtungsintensität) bei NeottiaSprossen keine Assimilation nachzuweisen ist.

\section{Feststellung des respiratorischen Quotienten}

Nachdem der Betrag der Förderung der Sauerstoffaufnahme pflanzlicher Gewebe in belichteten Lösungen fluoreszierender Substanzen bestimmt worden war, blieb noch die Frage nach der Art der oxydativen Prozesse zu klären, die durch diesen Effekt angeregt oder verstärkt wurden.

Die Möglichkeit, daß es sich bei der gesteigerten Sauerstoffaufnahme um eine Intensivierung der Atmung handeln könnte, war am einfachsten durch die Ermittelung des respiratorischen Quotienten zu prüfen, also des Verhältnisses $\mathrm{CO}_{2} / \mathrm{O}_{2}$ unter den Bedingungen, welche die vermehrte Sauerstoffaufnahme zur Folge hatten. Blieb hier der Quotient konstant, so mußte die gesamte Mehraufnahme zu einer Intensivierung der Atmung benutzt werden, während eine Verwendung des Sauerstoffes zu anderen oxydativen Prozessen, die nicht bis zur Bildung gasförmiger Endprodukte führen, eine Verschiebung des Quotienten bewirken mußte.

Die Bestimmung des respiratorischen Quotienten $(R Q)$ geschah in der Weise, daß in zwei Gefäße dieselbe Menge Material und eine Farbstofflösung derselben Konzentration kam, im einen aber mit Kalilauge absorbiert wurde, im anderen nicht. Manometer 1 zeigte daher den Sauerstoffverbrauch, Manometer 2 die Differenz zwischen Sauerstoffaufnahme und $\mathrm{CO}_{2}$-Abgabe an. Der $R Q$-Wert ergab sich aus der Formel:

$$
R Q=\frac{h_{1} K_{1}+h_{2} K_{2}}{h_{1} \cdot K_{1}} .
$$

( $h=$ Druckdifferenz in $\mathrm{mm}, K=$ Gefäßkonstante) .
Die Untersuchungen zeigten, daß der respiratorische Quotient bei Verwendung von Sproßgewebe von Asparagus officinalis und von Wurzelgewebe von Daucus Carota und Petroselinum hortense als Versuchsmaterial und von Fluoreszein-, Eosin- und Jodeosinlösungen (Konzentration jeweils $2,5 \times 10^{-4} \mathrm{~mol}$ ) als Versuchsmedien im Dunkeln wie im Licht durchweg den normalen Wert $(=1,0)$ aufwiesen. Die bei Belichtung pflanzlichen Gewebes in diesen Farbstoffen auftretende Intensivierung der Sauerstoffaufnahme dient also in vollem Umfange einer Steigerung der Atmung.

\section{Besprechung der Ergebnisse}

Die Erkenntnis, daß die durch fluoreszierende Farbstoffe katalysierte Lichtaktivierung des Sauerstoffes in pflanzlichen Geweben eine Steigerung der dissimilatorischen Vorgänge zur Folge hat, ist geeignet, die wichtigsten photodynamischen Wirkungen dem Verständnis näher zu bringen. Sowohl die Wachstumshemmung der belichteten Flanke von mit fluoreszierenden Farbstoffen behandelten Wurzeln, die dem „induzierten Phototropismus“ Metzners ${ }^{1}$ zugrunde liegt, als auch die Plasmaschädigungen, die sich bei Belichtung von Pflanzenteilen einstellen, die permeierte fluoreszierende Farbstoffe enthalten oder deren Chlorophyllfluoreszenz infolge einer Assimilationshemmung nicht mehr durch ihren Einbau in assimilatorische Prozesse „abgeschirmt“ ist, können als Folgen einer Atmungssteigerung aufgefaßt werden, die nicht von einer entsprechenden Förderung der Syntheseleistungen begleitet ist, also rein destruktiven Charakter trägt.

Die Tatsache, daß sich kein streng gesetzmäßiger Zusammenhang zwischen dem Betrag der Intensivierung und der bekannten Stärke der photodynamischen Wirkung der einzelnen Farbstoffe ergab, weist vielleicht darauf hin, daß es sich bei den Unterschieden in der photodynamischen Wirksamkeit weniger um die Folge einer verschieden intensiven Sauerstoffaktivierung als um die unterschiedlicher Permeations-, Absorptions- oder Speicherungsverhältnisse handelt. Die beschriebenen Versuche sind jedoch nicht umfassend genug, um hier eine klare Entscheidung treffen zu können; eine eingehendere Prüfung der Frage muß daher weiteren Untersuchungen vorbehalten bleiben.

14 F. W e b e r, Ber. dtsch. bot. Ges. 38, 233 [1920]; C. Montfort u. G. Küsters, Bot. Arch. 40, 571 [1940]; R. S c h a e d e, Die pflanzlichen Symbiosen, 2. Aufl., Jena 1948, S. 157. 